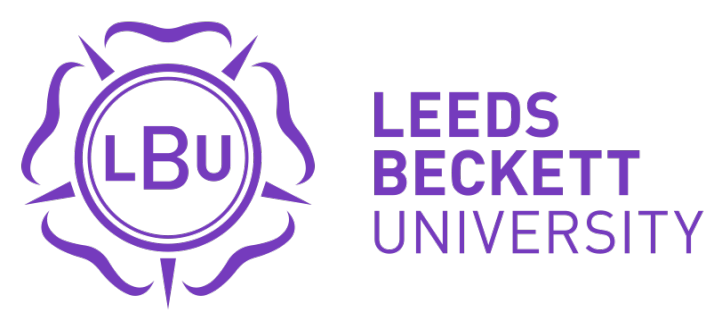

Citation:

o'Leary, T and Gifford, R and Double, R and Reynolds, R and Woods, D and Wardle, S and Greeves, $J$ (2019) Skeletal responses to an all-female unassisted Antarctic traverse. BONE, 121. pp. 267-276. ISSN 8756-3282 DOI: https://doi.org/10.1016/j.bone.2019.02.002

Link to Leeds Beckett Repository record:

https://eprints.leedsbeckett.ac.uk/id/eprint/5674/

Document Version:

Article (Accepted Version)

The aim of the Leeds Beckett Repository is to provide open access to our research, as required by funder policies and permitted by publishers and copyright law.

The Leeds Beckett repository holds a wide range of publications, each of which has been checked for copyright and the relevant embargo period has been applied by the Research Services team.

We operate on a standard take-down policy. If you are the author or publisher of an output and you would like it removed from the repository, please contact us and we will investigate on a case-by-case basis.

Each thesis in the repository has been cleared where necessary by the author for third party copyright. If you would like a thesis to be removed from the repository or believe there is an issue with copyright, please contact us on openaccess@leedsbeckett.ac.uk and we will investigate on a case-by-case basis. 


\section{Skeletal responses to an all-female unassisted Antarctic traverse}

Thomas J O’Leary ${ }^{\mathrm{a}}$, Robert M Gifford ${ }^{\mathrm{b}, \mathrm{c}}$, Rebecca L Double ${ }^{\mathrm{a}}$, Rebecca M Reynolds ${ }^{\mathrm{b}}$, David R Woods ${ }^{\mathrm{c}, \mathrm{d}, \mathrm{e}, \mathrm{f}}$, Sophie L Wardle ${ }^{\mathrm{a}}$, Julie P Greeves ${ }^{\mathrm{a}}$

${ }^{a}$ Army Personnel Research Capability, Army Headquarters, Andover, UK;

bUniversity/British Heart Foundation Centre for Cardiovascular Science, Queen's Medical Research Institute, University of Edinburgh, Edinburgh, UK; ${ }^{\mathrm{c}}$ Research and Clinical Innovation, Royal Centre for Defence Medicine, Birmingham, UK; ${ }^{\mathrm{d}}$ Research Institute for Sport, Physical Activity and Leisure, Leeds Beckett University, Leeds, UK; ${ }^{\mathrm{e}}$ Northumbria and Newcastle NHS Trusts, Wansbeck General and Royal Victoria Infirmary, Newcastle,

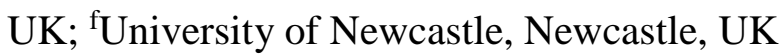

Author Email Addresses: Thomas J O’Leary, thomas.oleary100@mod.gov.uk; Robert M Gifford, r.gifford@ed.ac.uk; Rebecca L Double, rebecca.double102@mod.gov.uk; Rebecca M Reynolds, r.reynolds@ed.ac.uk; David R Woods, doctordrwoods@aol.com; Sophie L Wardle, sophie.arana104@mod.gov.uk; Julie P Greeves, julie.greeves143@mod.gov.uk

Corresponding author: Julie P Greeves OBE, Army Personnel Research Capability, Andover, Hampshire, United Kingdom, SP11 8HT. Email: julie.greeves143@ mod.gov.uk.

Running Title: Female bone health and extreme exercise 


\section{Abstract}

Purpose: To investigate the skeletal effects of the first all-female trans-Antarctic traverse. Methods: Six women (mean \pm SD, age $32 \pm 3$ years, height $1.72 \pm 0.07 \mathrm{~m}$, body mass $72.8 \pm$ $4.0 \mathrm{~kg}$ ) hauled $80 \mathrm{~kg}$ sledges over $1700 \mathrm{~km}$ in 61 days from coast-to-coast across the Antarctic. Whole-body areal bone mineral density (aBMD) (dual-energy X-ray absorptiometry) and tibial volumetric BMD (vBMD), geometry, microarchitecture and estimated mechanical properties (high-resolution peripheral quantitative computed tomography) were assessed 39 days before (pre-expedition) and 15 days after the expedition (post-expedition). Serum and plasma markers of bone turnover were assessed pre-expedition, and 4 and 15 days after the expedition. Results: There were reductions in trunk $(-2.6 \%)$, ribs $(-5.0 \%)$ and spine $(-3.4 \%)$ aBMD from pre- to post-expedition (all $\mathrm{P} \leq 0.046)$; arms, legs, pelvis and total body aBMD were not different (all $\mathrm{P} \geq 0.075$ ). Tibial vBMD, geometry, microarchitecture and estimated mechanical properties at the metaphysis (4\% site) and diaphysis $(30 \%$ site) were not different between pre- and post-expedition (all $\mathrm{P} \geq 0.082$ ). Bone-specific alkaline phosphatase was higher 15 days post- than 4 days post-expedition (1.7 $\left.\mu \mathrm{g} \cdot \mathrm{l}^{-1}, \mathrm{P}=0.028\right)$. Total $25(\mathrm{OH}) \mathrm{D}$ decreased from pre- to 4 days post-expedition $\left(-36 \mathrm{nmol} \cdot \mathrm{l}^{-}\right.$ $\left.{ }^{1}, \mathrm{P}=0.008\right)$. Sclerostin, procollagen $1 \mathrm{~N}$-terminal propeptide, C-telopeptide cross-links of type 1 collagen and adjusted calcium were unchanged (all $\mathrm{P} \geq 0.154$ ). Conclusion: A decline in $\mathrm{aBMD}$ of the axial skeleton may be due to indirect and direct effects of prolonged energy deficit. We propose that weight-bearing exercise was protective against the effects of energy deficit on tibial vBMD, geometry, microarchitecture and strength.

Keywords: bone density, bone microarchitecture, bone turnover, energy deficit, female athlete triad, polar expedition. 


\section{Highlights}

- We explored the effect of an unassisted Antarctic traverse on bone in women.

- The expedition reduced bone formation, but had no effect on bone resorption.

- The expedition reduced areal bone mineral density (BMD) in the axial, but not appendicular, skeleton.

- Tibial volumetric BMD, geometry, microarchitecture and strength were unaffected. 


\begin{abstract}
Abbreviations
25(OH)D, 25-hydroxyvitamin D; aBMD, areal bone mineral density; BALP, bone-specific alkaline phosphatase; CTX, c-telopeptide cross-links of type 1 collagen; DXA, dual energy X-ray absorptiometry; FHA, functional hypothalamic amenorrhoea; HPA, hypothalamic pituitary adrenal; HPG, hypothalamic pituitary gonadal; HR-pQCT, high-resolution peripheral quantitative computed tomography; IGF-1, insulin-like growth factor-1; P1NP, amino-terminal propeptide of procollagen type 1; T3, 3,3,5-triiodothyronine; vBMD, volumetric bone mineral density.
\end{abstract}




\section{Introduction}

Strenuous exercise can impair bone health in women, even in well-trained athletes [1-4]. Reduced energy availability (energy intake minus exercise energy expenditure) and low oestrogen are likely mechanisms for altered bone turnover and reduced areal bone mineral density (aBMD) [1-5]. Low energy availability exerts indirect effects on bone by suppressing the hypothalamic pituitary gonadal (HPG) axis (leading to functional hypothalamic amenorrhoea [FHA]) [1, 2, 6], decreasing oestradiol, and stimulating bone resorption [2], although oestradiol is only decreased in severe energy deficit [7]. This bone resorptive state decreases aBMD [8-11], and impairs bone microarchitecture and/or mechanical strength [8, $10,12,13]$, as shown in cross-sectional studies comparing amenorrhoeic and eumenorrhoeic athletes. The changes in bone quantity and quality that accompany FHA increase stress fracture risk [8]. Conversely, low energy availability exerts direct effects on bone through 3,3,5-triiodothyronine (T3), leptin and insulin-like growth factor-1 (IGF-1) mediated suppression of bone formation $[1,2,5,7]$. Prospective laboratory studies have shown reduced amino-terminal propeptide of procollagen type 1 (P1NP, bone formation maker) and increased c-telopeptide cross-links of type 1 collagen (CTX, bone resorption maker) in women with reductions in energy availability over several days [5, 7], although the long-term impact on bone density, geometry, microarchitecture and strength, has not been investigated.

The role of reduced energy availability on bone turnover is evident in military training. Bone formation decreased and bone resorption increased in male US Army Rangers who experienced energy deficits of between 1,000 to $4,000 \mathrm{kcal} \cdot \mathrm{d}^{-1}$ during 8 weeks of training [14], whereas bone formation and bone resorption increased after 8 weeks in female US Army recruits where energy provision was sufficient to meet the training requirements $[15$, 
16]. These anabolic changes in bone turnover in female recruits were accompanied by increased trabecular volumetric bone mineral density (vBMD), trabecular thickness, trabecular number and cortical thickness, and reduced cortical vBMD, increasing estimated bone strength under axial loading [15]. Measurements of bone macro- and microstructure were not taken in US Army Ranger training [14]. To our knowledge, no studies have prospectively investigated the effect of a prolonged period of severe exercise stress and energy deficit on bone turnover, density, geometry, microarchitecture and mechanical strength in women, who are more prone to stress fractures than men [17] and possibly more susceptible to metabolic perturbations associated with reduced energy availability [5]. A prospective approach would aid our understanding of the pathophysiology of bone injuries for women.

We have recently demonstrated resilient function of the HPG and hypothalamic pituitary adrenal (HPA) axes for the first all-female team to complete an unassisted crossing of Antarctica [18]. This extreme model of exercise represents a unique model to study skeletal health in response to high physiological stress and prolonged reduced energy availability. This study aimed to examine the skeletal responses to the first unassisted Antarctic traverse completed by an all-female team. We hypothesised that aBMD measured by DXA would decrease, particular in the trabecular axial skeleton, and microarchitecture and estimated mechanical strength in the tibia measured by high-resolution peripheral quantitative computed tomography (HR-pQCT) would deteriorate. We also hypothesised that bone turnover would uncouple, with increased bone resorption and suppressed bone formation.

\section{Methods}

\subsection{Participants}


Six women (mean $\pm \mathrm{SD}$, age $32 \pm 3$ years, height $1.72 \pm 0.07 \mathrm{~m}$, body mass $72.8 \pm 4.0 \mathrm{~kg}$ ) undertaking an unassisted coast-to-coast Antarctic ski expedition volunteered to take part in this study. The participants were the first all-female team to attempt this expedition and underwent 2 years of rigorous selection (from an original pool of 250 women) and training, including preparatory ski expeditions (full details can be found at http://exicemaiden.com/). All participants used hormonal contraceptives, with the intent to induce amenorrhoea during the expedition; one participant commenced levonorgestrel $150 \mathrm{mcg} /$ ethinylestradiol $30 \mathrm{mcg}$ immediately prior to the expedition whilst also using a progestogen-eluting Mirena ${ }^{\circledR}$ intrauterine device; one individual used progestogen-eluting Nexplanon ${ }^{\circledR}$ contraceptive implant; the other four participants used a progestogen-eluting Mirena ${ }^{\circledR}$ intrauterine device. Five participants were amenorrhoeic during the expedition and one menstruated twice within 4 - 10 days of due date, stating this was less frequent than normal. Each participant had the study procedures and risks explained to them before providing written informed consent. Ethical approval was received from the Ministry of Defence Research Ethics Committee (827/MoDREC/17).

\subsection{Study Design}

Each participant had whole-body aBMD, and tibial vBMD, geometry, microarchitecture and estimated mechanical strength, assessed 39 days before (pre-expedition) and 15 days after (36 hours after return to the UK [post-expedition]) an unassisted coast-to-coast Antarctic ski expedition. Additionally, blood samples were obtained after an overnight fast for the measurement of markers of bone turnover, 39 days before, and 4 and 15 days postexpedition. All measures were taken at the clinical research facility at the Royal Military Academy, Sandhurst, except for the blood sample 4 days post-expedition, which was taken in Punta Arenas, Chile (immediately after arrival from Antarctica). The expedition involved 
each individual hauling $80 \mathrm{~kg}$ sledges across $1700 \mathrm{~km}$ of the Antarctic and was completed in 61 days. For a full overview of the expedition, including dietary provision, see Gifford et al. [18].

\subsection{Whole-Body Areal Bone Mineral Density}

Whole-body aBMD was assessed using DXA (Lunar iDXA, GE Healthcare, UK) with participants wearing shorts and a t-shirt. Arms, legs, trunk, ribs, pelvis and spine aBMD were obtained from the whole-body scan. The changes in lean and fat mass have been reported previously [18].

\subsection{Tibial Volumetric Bone Mineral Density, Geometry and Microarchitecture}

A three-dimensional HR-pQCT system (XtremeCT II, Scanco Medical AG, Switzerland) was used to assess vBMD, geometry and microarchitecture of the tibia. A three-dimensional representation of approximately $10 \mathrm{~cm}$ of the right tibia in the axial direction, at both the metaphyseal (4\% site) and diaphyseal (30\% site) tibia, were obtained from 165 CT slices with an isotropic voxel size of $61 \mu \mathrm{m}$. Tibial length was measured before the first scan, taken as the distance between the medial malleolus and the tibial end plate. The leg of each participant was fitted into a carbon fibre shell and immobilised within the gantry of the scanner for the duration of the scan. A reference line was placed at the tibial endplate, with the first CT slice taken $4 \%$ and $30 \%$ of the tibia length from the reference line for the metaphyseal and diaphyseal tibia, respectively. For follow-up measurements at the $4 \%$ site, automatic algorithms matched the volumes of interest between baseline and follow-up scans, using the cross-sectional area within the periosteal boundary, so only the bone volume common to the baseline scans were assessed [19]. Of an initial 165 slices, on average $156 \pm 6$ slices were analysed on follow-up. The matching algorithms were disabled for analysis at the 
$30 \%$ site [15]. Daily quality control scans were performed using the manufacturer issued phantom that contained rods of hydroxyapatite (HA). The quality of each HR-pQCT scan was reviewed by a single operator and any scans judged to be of poor quality, as per the manufacturer visual grading of image quality, were excluded from the analyses; one baseline scan was re-performed due to movement artefact. The methods used to process the data have been previously described [19-21]. The standard evaluation procedure provided by the manufacturer was used to derive the following outcome variables: total vBMD $\left(\mathrm{mg} \mathrm{HA} \cdot \mathrm{cm}^{3}\right)$, trabecular vBMD $\left(\mathrm{mg} \mathrm{HA} \cdot \mathrm{cm}^{3}\right)$, cortical $\mathrm{vBMD}\left(\mathrm{mg} \mathrm{HA} \cdot \mathrm{cm}^{3}\right)$, trabecular bone volume fraction $(\%)$, trabecular area $\left(\mathrm{mm}^{2}\right)$, cortical area $\left(\mathrm{mm}^{2}\right)$, cortical thickness $(\mathrm{mm})$, trabecular thickness $(\mathrm{mm})$, trabecular number $\left(\mathrm{mm}^{-1}\right)$, trabecular separation $(\mathrm{mm})$, cortical porosity $(\%)$ and cortical pore diameter $(\mathrm{mm})$. Micro-finite element analysis was performed to calculate the biomechanical properties under uniaxial compression, specifically stiffness $\left(\mathrm{kN} \cdot \mathrm{mm}^{-1}\right)$ and failure load $(\mathrm{kN})$ [22]. Micro-finite element analysis failed for one post-exercise diaphyseal scan. All evaluations were performed by a single investigator to ensure consistency of periosteal and endosteal contouring. The coefficient of variation is $\leq 0.6 \%$ for $\mathrm{vBMD}, \leq 1.6 \%$ for trabecular microarchitecture and $1.9 \%$ for cortical thickness at the $4 \%$ site, and $\leq 0.3 \%$ for cortical and trabecular area, $\leq 0.1 \%$ for $\mathrm{vBMD}$ and $\leq 5 \%$ for cortical morphology at the $30 \%$ site [15].

\subsection{Biochemical Markers of Bone Turnover}

A venous blood sample was taken after an overnight fast for the measurement of bonespecific alkaline phosphatase (BALP), P1NP, sclerostin, CTX, total 25-hydroxyvitamin D (total 25(OH)D), magnesium, phosphate, calcium and albumin. Blood was collected in EDTA, serum-separating gel and fluoride oxalate tubes (Monovette ${ }^{\circledR}$, Sarstedt, Nümbrecht, Germany) and centrifuged at 5,000 rpm for 5 mins. Plasma and serum were stored at $-80^{\circ} \mathrm{C}$, 
or shipped to the UK on dry ice, prior to analysis. Analysis of BALP and sclerostin were performed by ELISA using proprietary kits (Quidel, Athens, $\mathrm{OH}$ and Biomedica Medizinprodukte GmbH, Vienna, Austria, respectively). P1NP and CTX were measured by Roche (®) Cobas e411 (Roche Diagnostics, Welwyn Garden City, UK) according to the manufacturer's instructions. Total 25(OH)D was measured using liquid chromatography/tandem mass spectrometry using automated solid phase extraction as described elsewhere [23]. Magnesium, phosphate, calcium and albumin were measured using commercial kits (Alpha Laboratories, Eastleigh, UK) adapted for use on a Cobas Fara centrifugal analyser. Inter-assay CV was $<10 \%$ for ELISAs and $<4 \%$ for e411 and Fara.

\subsection{Statistical Analysis}

All data were analysed using SPSS (v.24, SPSS Inc., USA) and initially checked for normality. Pre- to post-expedition changes in DXA and HR-pQCT outcomes are presented as mean absolute difference with $95 \%$ confidence intervals (95\% CI) and were compared using paired-sample t-tests. Non-parametric data (legs and trunk aBMD) were assessed with a Wilcoxon signed-rank test. Pre- to post-expedition changes in bone turnover markers are presented as mean absolute difference $(95 \% \mathrm{CI})$ between the pre-expedition and both postexpedition measurements ( 4 and 15 days post-expedition). Markers of bone turnover were assessed with a one-way repeated measures ANOVA (main effect of time [pre-expedition vs 4 days post-expedition vs 15 days post-expedition]), with post-hoc uncorrected t-tests used to assess differences between time-points in the event of a significant main effect. Nonparametric data (BALP, sclerostin, CTX and phosphate) were tested with a Friedman's test with the Wilcoxon-signed rank tests used for post-hoc analyses. Effect sizes are presented as Cohen's $d_{z}$ (mean difference divided by standard deviation of the mean difference) for 
paired-t-tests, with $0.20,0.50$ and 0.80 considered as small, medium and large, respectively. Significance was accepted as $\mathrm{P}<0.05$.

\section{Results}

\subsection{Whole-Body Areal Bone Mineral Density}

Areal $\mathrm{BMD}$ of the arms $(\mathrm{P}=0.632)$, legs $(\mathrm{P}=0.075)$, pelvis $(\mathrm{P}=0.137)$ and whole-body $(\mathrm{P}=$ 0.403) were not significantly different between pre- and post-expedition (Figure 1, Table 1). There was a significant reduction in trunk $(\mathrm{P}=0.046)$, ribs $(\mathrm{P}=0.007)$ and spine aBMD $(\mathrm{P}=$ 0.046) from pre- to post-expedition (Figure 1, Table 1).

\subsection{Volumetric Bone Mineral Density}

Total $(\mathrm{P}=0.306)$, trabecular $(\mathrm{P}=0.195)$ and cortical vBMD $(\mathrm{P}=0.172)$ of the tibial metaphysis (4\% site) were not significantly different between pre- and post-expedition (Figures $2 \mathrm{~A}-\mathrm{C}$, Table 1). Cortical vBMD $(\mathrm{P}=0.312)$ of the tibial diaphysis $(30 \%$ site) was not significantly different between pre- and post-expedition (Figure 3A, Table 1).

\subsection{Geometry}

Trabecular bone volume $(\mathrm{P}=0.835)$, trabecular area $(\mathrm{P}=0.422)$, cortical area $(\mathrm{P}=0.400)$, cortical thickness $(\mathrm{P}=0.082)$ and cortical perimeter $(\mathrm{P}=0.531$, data not shown $)$ of the tibial metaphysis (4\% site) were not significantly different between pre- and post-expedition (Figures 2D - G, Table 1). Trabecular area $(P=0.302)$, cortical area $(P=0.355)$, cortical thickness $(\mathrm{P}=0.384)$ and cortical perimeter $(\mathrm{P}=0.392)$ of the tibial diaphysis $(30 \%$ site $)$ were not significantly different between pre- and post-expedition (Figures 3B - E, Table 1).

\subsection{Microarchitecture}


Trabecular number $(\mathrm{P}=0.573)$, trabecular thickness $(\mathrm{P}=0.616)$, trabecular spacing $(\mathrm{P}=$ 0.416), cortical pore diameter $(\mathrm{P}=0.903)$ and cortical porosity $(\mathrm{P}=0.474)$ for the tibial metaphysis (4\% site) were not significantly different between pre- and post-expedition (Figure $2 \mathrm{H}-\mathrm{L}$, Table 1$)$. Cortical pore diameter $(\mathrm{P}=0.492)$ and cortical porosity $(\mathrm{P}=0.679)$ for the tibial diaphysis ( $30 \%$ site) were not significantly different between pre- and postexpedition (Figures 3F - G, Table 1).

\subsection{Mechanical Strength}

Stiffness $(\mathrm{P}=0.664)$ and failure load $(\mathrm{P}=0.688)$ for the tibial metaphysis $(4 \%$ site $)$, and stiffness $(\mathrm{P}=0.829)$ and failure load $(\mathrm{P}=0.403)$ for the tibial diaphysis $(30 \%$ site $)$ were not significantly different between pre- and post-expedition (Figures 4A - D, Table 1). 

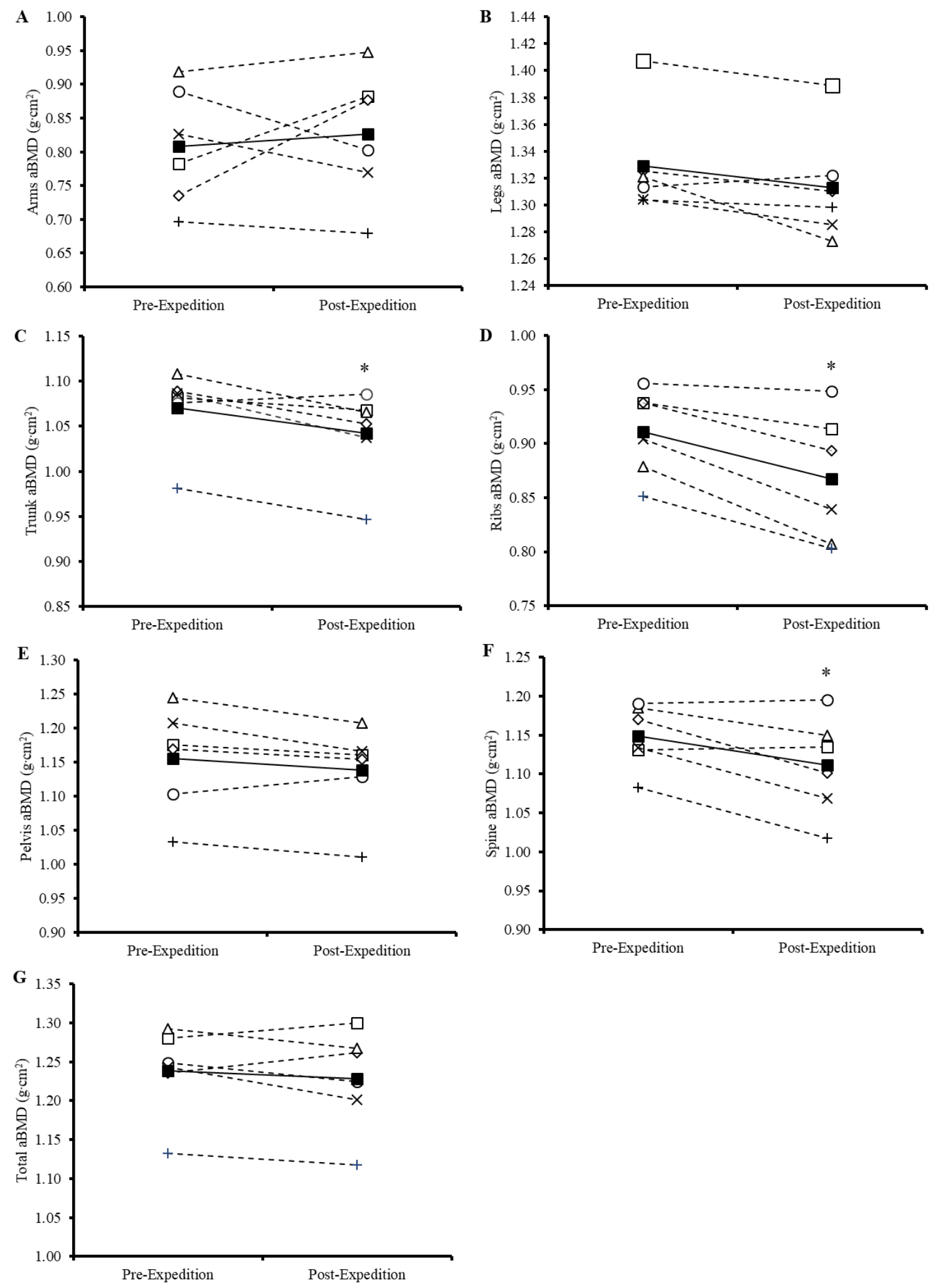

Figure 1. Whole-body and regional areal bone mineral density before and after the expedition. Solid line represents mean data; dashed lines are individual participants, each represented by a different symbol.

$* \mathrm{P}<0.05$ vs pre-expedition; aBMD, areal bone mineral density. 

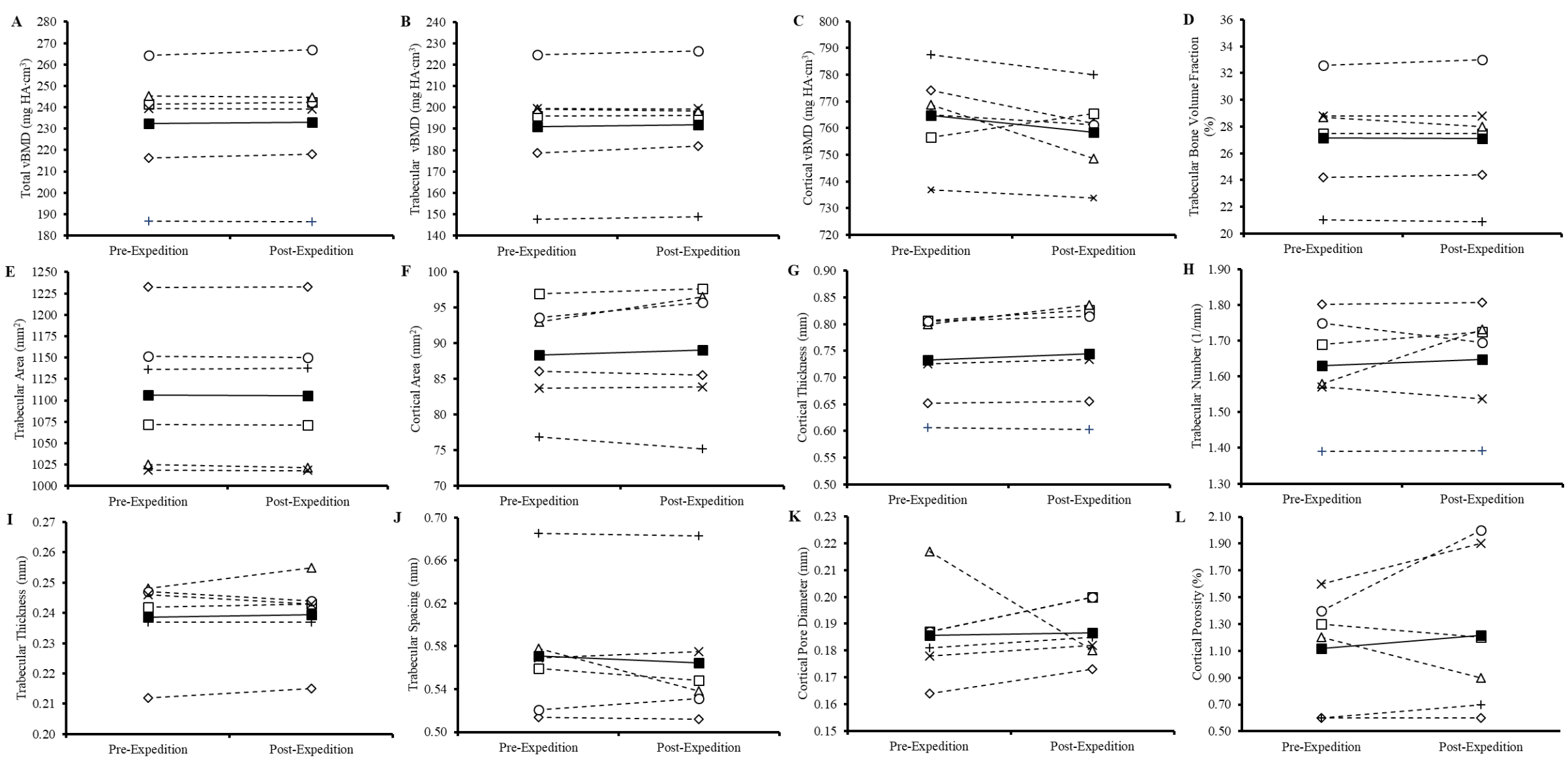

Figure 2. Volumetric bone mineral density (A - C), geometry (D - G) and microarchitecture (H - L) of the tibial metaphysis (4\% site) before and after the expedition. Solid line represents mean data; dashed lines are individual participants, each represented by a different symbol. $\mathrm{mg} \mathrm{HA} \cdot \mathrm{cm}^{3}$, milligram of hydroxyapatite per cubic centimetre; vBMD, volumetric bone mineral density. 

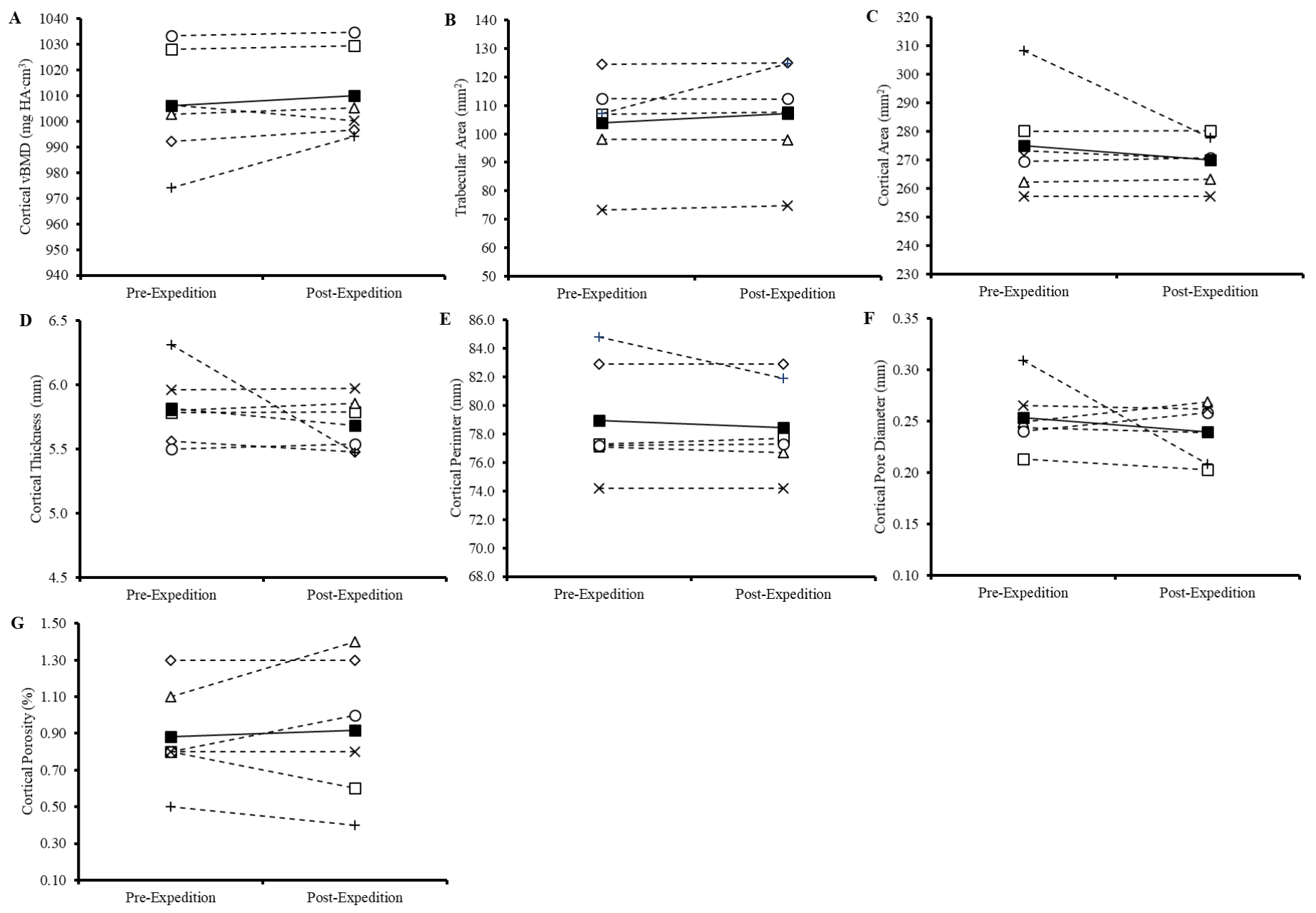

Figure 3. Volumetric bone mineral density (A), geometry (B - E) and microarchitecture (F - G) of the tibial diaphysis (30\% site) before and after the expedition. Solid line represents mean data; dashed lines are individual participants, each represented by a different symbol. mg HA $\cdot \mathrm{cm}^{3}$, milligram of hydroxyapatite per cubic centimetre; $\mathrm{vBMD}$, volumetric bone mineral density 

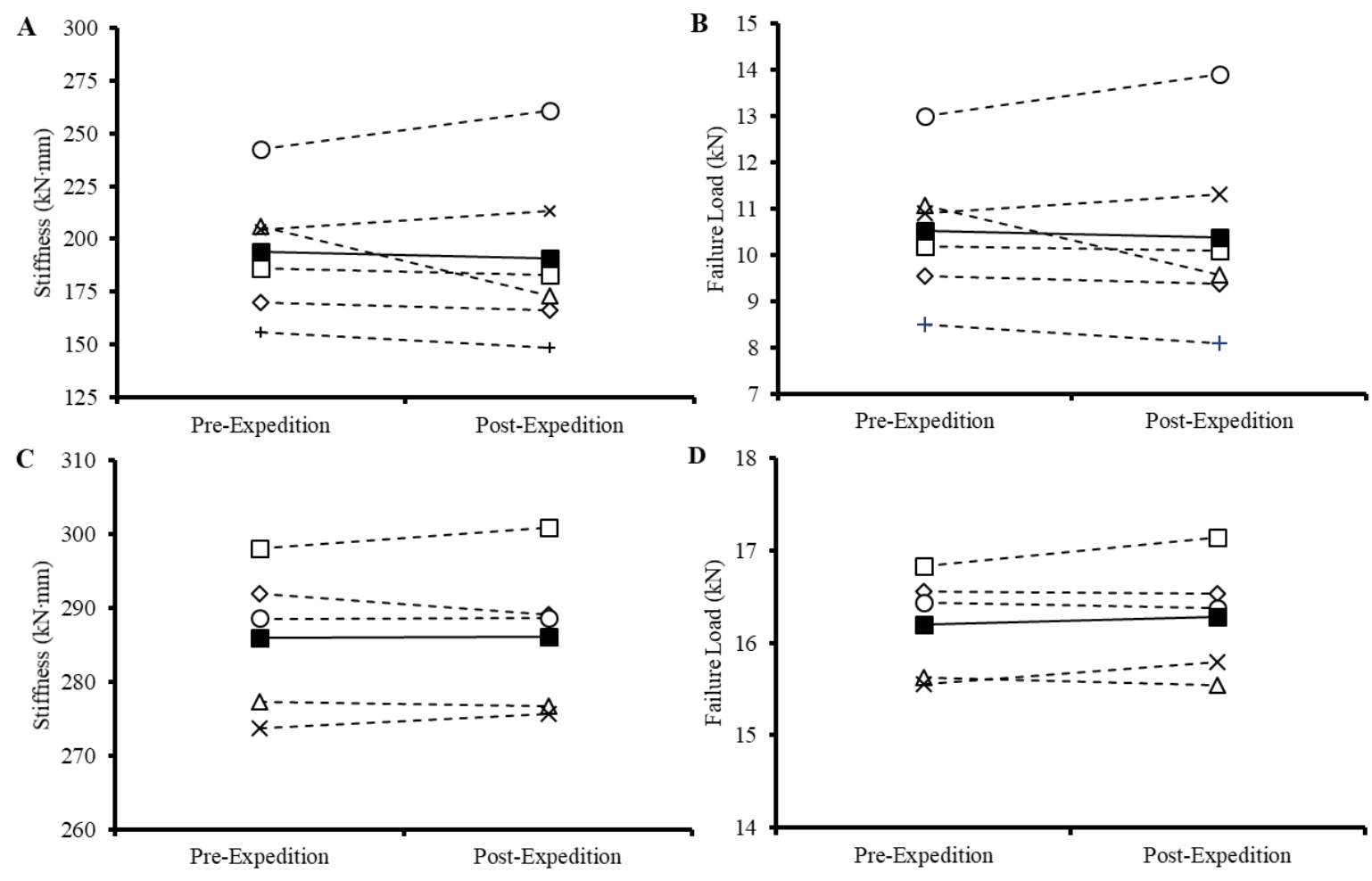

Figure 4. Stiffness and failure load for the tibial metaphysis (4\% site) (A and B) and tibial diaphysis (30\% site) (C and D) before and after the expedition. Solid line represents mean data; dashed lines are individual participants, each represented by a different symbol. $\mathrm{kN}$, kilonewtons; $\mathrm{kN} \cdot \mathrm{mm}$, kilonewtons per millimetre 
Table 1. Change in dual-energy X-ray absorptiometry and high-resolution peripheral quantitative computed tomography outcomes from pre-expedition to post-expedition.

Pre-expedition vs Post-expedition

Mean Difference (95\% CI)

$\operatorname{aBMD}\left(\mathrm{g} \cdot \mathrm{cm}^{2}\right)$

Arms

Legs

Trunk

Ribs

Pelvis

Spine

Whole-body

Tibial Metaphysis (4\% site)

Total vBMD $\left(\mathrm{mg} \mathrm{HA} \cdot \mathrm{cm}^{3}\right)$
Trabecular vBMD $\left(\mathrm{mg} \mathrm{HA} \cdot \mathrm{cm}^{3}\right)$
Cortical vBMD $\left(\mathrm{mg} \mathrm{HA} \cdot \mathrm{cm}^{3}\right)$
Trabecular Bone Volume $(\%)$
Trabecular Area $\left(\mathrm{mm}^{2}\right)$
Cortical Area $\left.(\mathrm{mm})^{2}\right)$
Cortical Thickness $(\mathrm{mm})$
Cortical Perimeter $(\mathrm{mm})$
Trabecular Number $(1 \cdot \mathrm{mm})$
Trabecular Thickness $(\mathrm{mm})$
Trabecular Spacing $(\mathrm{mm})$
Cortical Pore Diameter $(\mathrm{mm})$
Cortical Porosity $(\%)$
Stiffness ( $\mathrm{kN} \cdot \mathrm{mm})$

Failure Load (kN)

Tibial Diaphysis (30\% site) $d_{z} \quad P$

$\begin{array}{ll}0.21 & 0.632 \\ -0.87 & 0.075 \\ -1.27 & \mathbf{0 . 0 4 6} \\ -1.80 & \mathbf{0 . 0 0 7} \\ -0.72 & 0.137 \\ -1.08 & \mathbf{0 . 0 4 6} \\ -0.37 & 0.403\end{array}$

\begin{tabular}{|c|c|c|}
\hline $0.6(-0.7,2.0)$ & 0.47 & 0.306 \\
\hline $0.9(-0.6,2.4)$ & 0.61 & 0.195 \\
\hline$-6.4(-16.6,3.9)$ & -0.65 & 0.172 \\
\hline$-0.03(-0.42,0.36)$ & -0.09 & 0.835 \\
\hline$-0.65(-2.56,1.26)$ & -0.36 & 0.422 \\
\hline $0.70(-1.26,2.66)$ & 0.92 & 0.400 \\
\hline $0.012(-0.002,0.027)$ & 0.89 & 0.082 \\
\hline $0.2(-0.6,1.0)$ & 0.27 & 0.531 \\
\hline $0.02(-0.06,0.09)$ & 0.25 & 0.573 \\
\hline $0.001(-0.003,0.005)$ & 0.22 & 0.616 \\
\hline$-0.007(-0.025,0.012)$ & -0.36 & 0.416 \\
\hline $0.001(-0.019,0.021)$ & 0.05 & 0.903 \\
\hline $0.10(-0.23,0.43)$ & 0.32 & 0.474 \\
\hline$-3.2(-21.3,14.8)$ & -0.19 & 0.664 \\
\hline$-0.1(-1.0,0.7)$ & -0.17 & 0.688 \\
\hline
\end{tabular}

.306

.195

.172

0.835

.422

.400

.082

0.531

0.573

.616

0.416

.903

0.474

664

0.688 


\begin{tabular}{lccc}
\hline Cortical $v B M D\left(\mathrm{mg} \mathrm{HA} \cdot \mathrm{cm}^{3}\right)$ & $4.0(-5.1,13.1)$ & 0.46 & 0.312 \\
Trabecular Area $\left(\mathrm{mm}^{2}\right)$ & $3.3(-4.0,10.6)$ & 0.47 & 0.302 \\
Cortical Area $\left(\mathrm{mm}^{2}\right)$ & $-5.2(-18.3,7.9)$ & -0.42 & 0.355 \\
Cortical Thickness $(\mathrm{mm})$ & $-0.14(-0.50,0.23)$ & -0.39 & 0.384 \\
Cortical Perimeter $(\mathrm{mm})$ & $-0.5(-1.7,0.8)$ & -0.38 & 0.392 \\
Cortical Pore Diameter $(\mathrm{mm})$ & $-0.014(-0.060,0.033)$ & -0.30 & 0.492 \\
Cortical Porosity $(\%)$ & $0.03(-0.16,0.23)$ & 0.18 & 0.679 \\
Stiffness $(\mathrm{kN} \cdot \mathrm{mm})$ & $0.2(-2.5,2.9)$ & 0.10 & 0.829 \\
Failure Load $(\mathrm{kN})$ & $0.1(-0.1,0.3)$ & 0.42 & 0.403
\end{tabular}

aBMD, areal bone mineral density; $\mathrm{kN}$, kilonewtons; $\mathrm{kN} \cdot \mathrm{mm}$, kilonewtons per millimetre; $\mathrm{mg} \mathrm{HA} \cdot \mathrm{cm}^{3}$, milligram of hydroxyapatite per cubic centimetre; vBMD, volumetric bone mineral density.

\subsection{Biochemical Markers of Bone Turnover}

All bone turnover data are presented in Figure 5 with the pre- to post-expedition changes shown in Table 2. There was a significant effect of time for BALP $(P=0.042)$. Post-hoc analysis revealed that BALP was higher 15 days post-expedition than 4 days post-expedition $(P=0.028)$. There was a significant effect of time for phosphate $(P=0.030)$. Post-hoc analysis revealed that phosphate was higher 15 days post-expedition than pre-expedition $(\mathrm{P}=$ $0.028)$ and 4 days post-expedition $(\mathrm{P}=0.046)$. There was no significant effect of time for sclerostin, P1NP, CTX, magnesium or albumin-adjusted calcium $(\mathrm{P}=0.154-0.846)$. There was a significant reduction in total $25(\mathrm{OH}) \mathrm{D}$ from pre- to 4 days post-expedition $(\mathrm{P}=0.008)$. 

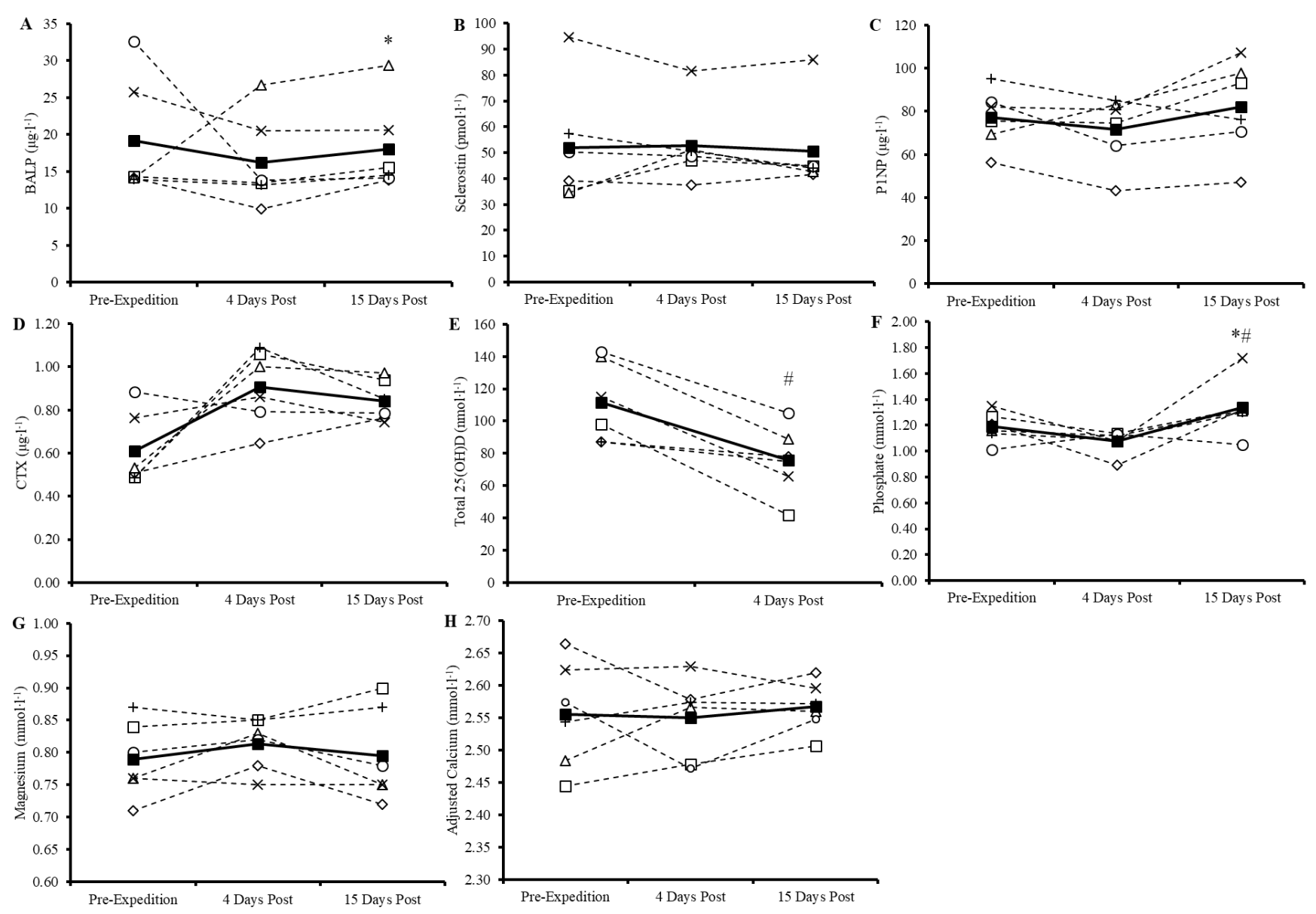

Figure 5. Markers of bone turnover before and after the expedition. Solid line represents mean data; dashed lines are individual participants, each represented by a different symbol.

BALP, bone-specific alkaline phosphatase; CTX, C-telopeptide cross-links of type 1 collagen; P1NP, procollagen 1 N-terminal propeptide; Total 25(OH)D, total vitamin D. ${ }^{\#} \mathrm{P}<0.05$ vs Pre-Expedition; *P $<0.05$ vs 4 Days Post 
Table 2. Change in bone turnover markers from pre-expedition to 4 days and 15 days postexpedition.

\begin{tabular}{|c|c|c|c|c|}
\hline & \multicolumn{2}{|c|}{ Pre- vs 4 days post-expedition } & \multicolumn{2}{|c|}{ Pre- vs 15 days post-expedition } \\
\hline & Mean Difference (95\% CI) & $d_{z}$ & Mean Difference (95\% CI) & $d_{z}$ \\
\hline $\operatorname{BALP}\left(\mu \mathrm{g} \cdot 1^{-1}\right)$ & $-2.9(-13.4,7.7)$ & -0.29 & $-1.1(-12.6,10.3)$ & -0.11 \\
\hline Sclerostin $\left(\mathrm{pmol} \cdot \mathrm{l}^{-1}\right)$ & $1(-11,13)$ & 0.07 & $-1(11,9)$ & -0.08 \\
\hline $\operatorname{P} 1 \mathrm{NP}\left(\mu \mathrm{g} \cdot \mathrm{l}^{-1}\right)$ & $-5(-18,7)$ & -0.46 & $5(-17,27)$ & 0.23 \\
\hline $\operatorname{CTX}\left(\mu \mathrm{g} \cdot 1^{-1}\right)$ & $0.30(-0.01,0.60)$ & 1.03 & $0.23(-0.02,0.48)$ & 0.97 \\
\hline Total 25(OH)D $\left(\mathrm{nmol} \cdot \cdot^{-1}\right)$ & $-36(-57,-14)^{*}$ & -1.75 & - & - \\
\hline Phosphate $\left(\mathrm{mmol} \cdot \mathrm{l}^{-1}\right)$ & $-0.12(-0.29,0.06)$ & -0.71 & $0.15(0.02,0.27)^{*}$ & 1.25 \\
\hline Magnesium $\left(\mathrm{mmol} \cdot 1^{-1}\right)$ & $0.02(-0.02,0.06)$ & 0.60 & $0.01(-0.03,0.04)$ & 0.17 \\
\hline Adjusted Calcium $\left(\mathrm{mmol} \cdot \cdot^{-1}\right)$ & $-0.01(-0.08,0.07)$ & -0.08 & $0.01(-0.04,0.06)$ & 0.22 \\
\hline
\end{tabular}

\section{Discussion}

We present findings from the first observational study examining the skeletal effects of an unassisted Antarctic traverse completed by women. We observed reductions in trunk, ribs and spine aBMD from pre- to post-expedition. Volumetric BMD, geometry, microarchitecture and estimated mechanical strength at the tibial metaphysis (4\% site) and diaphysis (30\% site) were unchanged. We also showed a decrease in markers of bone formation (BALP). The participants were the first all-female team to complete an unassisted Antarctic traverse and, therefore, combined with the use of imaging techniques (DXA and HR-pQCT) and markers of bone turnover, this study offers a novel and comprehensive overview of skeletal adaptations to an extreme model of physiological stress in women. Evidence for the long-term effects of reduced energy availability on bone health in women is 
mostly provided by cross-sectional studies [5]; here we present, to our knowledge, the first longitudinal study examining changes in bone turnover, density, geometry, microarchitecture and estimated mechanical strength in response to a prolonged energy deficit for women.

\subsection{Whole-Body Areal Bone Mineral Density}

We showed reductions in trunk $(-2.6 \%)$, ribs $(-5.0 \%)$ and spine $(-3.4 \%)$ aBMD from pre- to post-expedition. Albeit non-significant, we also observed large and moderate effect sizes for reductions in legs $(-1.2 \%)$ and pelvis $(-1.5 \%)$ aBMD, respectively. Participants lost on average $9.4 \pm 2.3 \mathrm{~kg}$ of body mass $(-13 \%)$, comprising mostly body fat, and had marked reductions in leptin, a marker of energy status, demonstrating reduced energy availability despite the careful dietary planning (provision of $4990 \mathrm{kcal} \cdot \mathrm{d}^{-1}$ ) [18]. Low energy availability impairs bone indirectly by suppressing the HPG axis leading to FHA [1, 2, 6]. Using a novel dynamic assessment of the HPG axes, we have demonstrated largely preserved HPG axis function in these women, but showed lower oestradiol at 4 days compared to 15 days postexpedition, which might indicate an immediate post-expedition suppression with a subsequent recovery [18]. Oestrogen suppresses osteoclast activity [24] and, therefore, low oestrogen increases bone resorption $[2,7]$, but only when the energy deficit is severe enough [7]. The apparent increase in CTX (non-significant but large effect sizes) 4 and 15 days postexpedition compared with pre-expedition supports a prolonged increase in bone resorption and may explain the reductions in axial aBMD. In support of these findings, cross-sectional studies have demonstrated that amenorrhoeic athletes have lower spine (5 - 13\%) [9-11], pelvis $(6-13 \%)$ [9-11], trunk $(\sim 10 \%)$ [11] and femoral neck aBMD [8] than eumenorrheic athletes, and lower ribs aBMD ( 9\%) than eumenorrheic controls [11]. 
Low energy availability directly impairs bone turnover and bone mass $[1,5,9,13]$, through effects on cortisol, T3, leptin and IGF-1 [1, 2, 5, 25]. We previously reported preserved thyroid function but reduced leptin following the expedition [18]. Leptin influences bone turnover directly through receptors on osteoblasts, and indirectly though effects on oestrogen, cortisol, IGF-1 and parathyroid hormone $(\mathrm{PTH})$ [26]. Alongside reductions in leptin, we found elevated hair cortisol and lower IGF-1 at 4 days post-expedition compared to 15 day post-expedition, indicating a possible suppression [18]. These hormonal alterations, which suppress bone formation [2], may explain decreased BALP at 4 compared with 15 days postexpedition, and may have contributed to the reductions in aBMD. The effect of low energy availability on aBMD may have also been exacerbated in the presence of low oestradiol [13, 27]. In agreement with our data, suppression of BALP [14], and increases in cortisol and reductions in IGF-1 [28], have been reported following 8 weeks US Army Ranger training for men. Conversely, the increase in BALP in female recruits following US Army basic training $[15,16]$ is likely due to differences in energy balance between studies. We postulate that the severity and duration of energy deficit in our study was more similar to US Army Ranger training than US Army basic training, as evidenced by similar losses in body mass $(-13 \%)$ over a similar duration (62 days). The reduction in spinal aBMD we observed is greater than the 1 to $2 \%$ reductions in lumbar spine aBMD reported following 15 weeks of Swiss Army basic military training [29] and an Antarctic traverse [30], and occurred at about twice the rate of space travel ( -0.9 to $-1 \%$ per month) [31], all in men. Total $25(\mathrm{OH}) \mathrm{D}$ also decreased following the expedition, which could impair bone by increasing PTH [14, 16, 32-34], although post-expedition total $25(\mathrm{OH}) \mathrm{D}$ may have been sufficient to prevent aBMD loss [33]. An increase in PTH may also explain the lower phosphate at 4 compared with 15 days postexpedition, but, PTH was not measured in this study. The reduction in aBMD we report is likely due to the indirect and/or direct effects of reduced energy availability on bone turnover 
$[1,2,4,6]$, which has not been well studied longitudinally in women. Anatomical sites with high trabecular content, or with reduced loading, are particularly responsive to low energy availability [4], which likely explains the losses in aBMD in the axial, but preservation of bone in appendicular skeleton. The mechanical loading profile of the expedition remains unknown, however skiing appears osteogenic for the lower limbs [35].

\subsection{Tibial Volumetric Bone Mineral Density}

There were no differences in total, trabecular or cortical vBMD at either the metaphyseal or diaphyseal sites between pre- and post-expedition. Although non-significant, there were moderate effect sizes for increases in trabecular vBMD $(0.5 \%)$ and reductions in cortical vBMD $(-0.8 \%)$ at the metaphyseal site, which is consistent with mechanical loading [15] but not low energy availability [13]. Hughes et al. [15] recently observed an increase in trabecular vBMD $(2 \%)$ and reduction in cortical vBMD $(-0.3 \%)$ at the tibial metaphysis for women undergoing basic military training. An increase in trabecular vBMD is recognised as an early adaptation to mechanical loading [15, 36-39], whereas a reduction in cortical vBMD could be due to the unmineralised nature of new bone $[10,15]$. Basic military training is characterised by large increases in physical [40-42] and locomotor activity [40, 43], and therefore the mechanical loading for the tibia is considered to be high [15, 38, 44, 45]. Alpine skiing, the closest model of exercise to polar exploration, appears to increase trabecular vBMD of the tibia [35], but the mechanical loading profile of the expedition was not studied and each participant had undergone two years of prior training. Preservation of BMD at the appendicular, but not axial skeleton, is consistent with cross-sectional studies demonstrating similar trabecular and cortical vBMD at the distal tibia between amenorrhoeic and eumenorrhoeic athletes $[10,12]$, and the known negative effects of low energy availability on trabecular bone, particular at non-weight bearing sites [4]. These data suggest mechanical 
loading was protective for trabecular vBMD and may reduce cortical vBMD during low energy availability.

\subsection{Tibial Geometry, Microarchitecture and Mechanical Strength}

There were no differences in tibial geometry, microarchitecture, or estimated mechanical properties at the metaphysis or diaphysis between pre- and post-expedition. Whilst trabecular microarchitecture may be impaired at the distal tibia in amenorrhoeic compared with eumenorrhoeic athletes (lower trabecular number and greater trabecular spacing) [10], differences in cortical and trabecular geometry, and microarchitecture between groups are not always reported $[8,12]$. Trabecular bone is more susceptible to the deleterious effects of low energy availability [4], but studies also report decreased cortical area and thickness [13]. Adaptations to the trabecular microarchitecture (increased trabecular number and thickness, and reduced spacing), and increased cortical thickness $(1.0 \%)$, contribute to improved mechanical strength following US Army basic training for women [15]. Albeit nonsignificant, large effect sizes were detected for increases in cortical thickness $(1.7 \%)$ and cortical area $(0.8 \%)$ and at the metaphysis, consistent with mechanical loading. It is therefore possible that the lack of change in trabecular microarchitecture and estimated mechanical strength were due to mechanical loading experienced as part of the expedition, negating any deleterious effects of reduced energy availability. These observations further support the notion that mechanical loading preserves lower limb bone quality (trabecular microarchitecture, cortical geometry and estimated mechanical strength), which could be important in protecting against lower limb stress fracture risk [8].

\subsection{Limitations}


The main limitation to our study is the low number of participants reducing statistical power, and the data must be interpreted with this consideration. Small samples are typical of extreme expeditions, but exposure to the exercise, nutrition and sleep were similar between participants, and this study provides novel insight into the effects of exposure to extreme exercise and energy deficit. Another limitation is the delay between testing before and after the expedition as we were unable to take measurements in Antarctica; follow up measurements after the expedition were made as soon as practicable, in both Chile and on return to the U.K. Finally, we did not assess the microarchitecture and mechanical strength in a non-weight-bearing bone, which may have revealed changes to bone caused by low energy availability, independent of changes in mechanical loading.

\subsection{Conclusion}

The first all-female team to complete an unassisted Antarctic traverse experienced reductions in trunk, ribs and spine aBMD, and altered bone turnover. These impairments in bone turnover and aBMD occurred in women taking hormonal contraceptives, which we propose are the result of prolonged periods of low energy availability. Tibial vBMD, geometry and microarchitecture, of both trabecular and cortical bone, and estimated mechanical strength, at the metaphysis (4\% site) and diaphysis (30\% site) were not significantly affected. It is likely the mechanical loading of the lower limbs protected tibial bone against the deleterious effect of prolonged energy deficit.

\section{Acknowledgements}

The authors are indebted to the Expedition ICE MAIDEN team for their participation in a very challenging study. We are grateful to the Wellcome Trust Clinical Research Facility, and particularly Jo Singleton, for help with data collection. We thank Antarctic Logistic 
Expeditions Ltd. for supporting researchers in Antarctica and Chile. The authors would also like to thank Miss Sally Handford and Miss Jennifer Wright for assisting with data collection, and Dr Fiona Koivula for critical review of the manuscript.

\section{Funding}

The study was funded by the UK Ministry of Defence.

\section{Competing Interests}

The authors have no competing interests to declare.

\section{References}

[1] M. Mountjoy, J. Sundgot-Borgen, L. Burke, S. Carter, N. Constantini, C. Lebrun, N. Meyer, R. Sherman, K. Steffen, R. Budgett, A. Ljungqvist, The IOC consensus statement: beyond the Female Athlete Triad-Relative Energy Deficiency in Sport (RED-S), Br J Sports Med 48(7) (2014) 491-7.

[2] A. Nattiv, A.B. Loucks, M.M. Manore, C.F. Sanborn, J. Sundgot-Borgen, M.P. Warren, M. American College of Sports Medicine Position Stand. The female athlete triad, Med Sci Sports Exerc 39(10) (2007) 1867-82.

[3] M.J. De Souza, A. Nattiv, E. Joy, M. Misra, N.I. Williams, R.J. Mallinson, J.C. Gibbs, M. Olmsted, M. Goolsby, G. Matheson, Expert Panel, 2014 Female Athlete Triad Coalition Consensus Statement on Treatment and Return to Play of the Female Athlete Triad: 1st International Conference held in San Francisco, California, May 2012 and 2nd International Conference held in Indianapolis, Indiana, May 2013, Br J Sports Med 48(4) (2014) 289.

[4] M. Mountjoy, J. Sundgot-Borgen, L. Burke, K.E. Ackerman, C. Blauwet, N. Constantini, C. Lebrun, B. Lundy, A. Melin, N. Meyer, R. Sherman, A.S. Tenforde, M.K. Torstveit, R. 
Budgett, International Olympic Committee (IOC) Consensus Statement on Relative Energy Deficiency in Sport (RED-S): 2018 Update, Int J Sport Nut Exerc Metab 28(4) (2018) 316331.

[5] M. Papageorgiou, E. Dolan, K.J. Elliott-Sale, C. Sale, Reduced energy availability: implications for bone health in physically active populations, Eur J Nutr 57(3) (2018) 847859.

[6] R.M. Gifford, R.M. Reynolds, J. Greeves, R.A. Anderson, D.R. Woods, Reproductive dysfunction and associated pathology in women undergoing military training, J R Army Med Corps 163(5) (2017) 301-310.

[7] R. Ihle, A.B. Loucks, Dose-response relationships between energy availability and bone turnover in young exercising women, J Bone Miner Res 19(8) (2004) 1231-40.

[8] K.E. Ackerman, N. Cano Sokoloff, D.E.N.M. G, H.M. Clarke, H. Lee, M. Misra, Fractures in Relation to Menstrual Status and Bone Parameters in Young Athletes, Med Sci Sports Exerc 47(8) (2015) 1577-86.

[9] K.L. Cobb, L.K. Bachrach, G. Greendale, R. Marcus, R.M. Neer, J. Nieves, M.F. Sowers, B.W. Brown, Jr., G. Gopalakrishnan, C. Luetters, H.K. Tanner, B. Ward, J.L. Kelsey, Disordered eating, menstrual irregularity, and bone mineral density in female runners, Med Sci Sports Exerc 35(5) (2003) 711-9.

[10] K.E. Ackerman, T. Nazem, D. Chapko, M. Russell, N. Mendes, A.P. Taylor, M.L. Bouxsein, M. Misra, Bone microarchitecture is impaired in adolescent amenorrheic athletes compared with eumenorrheic athletes and nonathletic controls, J Clin Endocrinol Metab 96(10) (2011) 3123-33.

[11] J. Piasecki, A. Ireland, M. Piasecki, J. Cameron, J.S. McPhee, H. Degens, The strength of weight-bearing bones is similar in amenorrheic and eumenorrheic elite long-distance runners, Scand J Med Sci Sports 28(5) (2018) 1559-1568. 
[12] K.E. Ackerman, M. Putman, G. Guereca, A.P. Taylor, L. Pierce, D.B. Herzog, A. Klibanski, M. Bouxsein, M. Misra, Cortical microstructure and estimated bone strength in young amenorrheic athletes, eumenorrheic athletes and non-athletes, Bone 51(4) (2012) 6807.

[13] E.A. Southmayd, R.J. Mallinson, N.I. Williams, D.J. Mallinson, M.J. De Souza, Unique effects of energy versus estrogen deficiency on multiple components of bone strength in exercising women, Osteoporos Int 28(4) (2017) 1365-1376.

[14] J.M. Hughes, M.A. Smith, P.C. Henning, D.E. Scofield, B.A. Spiering, J.S. Staab, J.R. Hydren, B.C. Nindl, R.W. Matheny, Jr., Bone formation is suppressed with multi-stressor military training, Eur J Appl Physiol 114(11) (2014) 2251-9.

[15] J.M. Hughes, E. Gaffney-Stomberg, K.I. Guerriere, K.M. Taylor, K.L. Popp, C. Xu, G. Unnikrishnan, J.S. Staab, R.W. Matheny, Jr., J.P. McClung, J. Reifman, M.L. Bouxsein, Changes in tibial bone microarchitecture in female recruits in response to 8 weeks of U.S. Army Basic Combat Training, Bone 113 (2018) 9-16.

[16] L.J. Lutz, J.P. Karl, J.C. Rood, S.J. Cable, K.W. Williams, A.J. Young, J.P. McClung, Vitamin D status, dietary intake, and bone turnover in female Soldiers during military training: a longitudinal study, J Int Soc Sports Nut 9(1) (2012) 38.

[17] L. Wentz, P.Y. Liu, E. Haymes, J.Z. Ilich, Females have a greater incidence of stress fractures than males in both military and athletic populations: a systemic review, Mil Med 176(4) (2011) 420-30.

[18] R.M. Gifford, T. O'Leary, R. Cobb, J. Blackadder-Weinstein, R. Double, S.L. Wardle, R.A. Anderson, C. Doug Thake, J. Hattersley, C. Imray, A. Wilson, J.P. Greeves, R.M. Reynolds, D.R. Woods, Female Reproductive, Adrenal, and Metabolic Changes during an Antarctic Traverse, Med Sci Sports Exerc (in press). 
[19] S. Boutroy, M.L. Bouxsein, F. Munoz, P.D. Delmas, In vivo assessment of trabecular bone microarchitecture by high-resolution peripheral quantitative computed tomography, J Clin Endocrinol Metab 90(12) (2005) 6508-15.

[20] A.J. Burghardt, H.R. Buie, A. Laib, S. Majumdar, S.K. Boyd, Reproducibility of direct quantitative measures of cortical bone microarchitecture of the distal radius and tibia by HRpQCT, Bone 47(3) (2010) 519-28.

[21] A.J. Burghardt, G.J. Kazakia, S. Ramachandran, T.M. Link, S. Majumdar, Age- and gender-related differences in the geometric properties and biomechanical significance of intracortical porosity in the distal radius and tibia, J Bone Miner Res 25(5) (2010) 983-93.

[22] N. Vilayphiou, S. Boutroy, P. Szulc, B. van Rietbergen, F. Munoz, P.D. Delmas, R. Chapurlat, Finite Element Analysis Performed on Radius and Tibia HR-pQCT Images and Fragility Fractures at All Sites in Men, J Bone Miner Res 26(5) (2011) 965-73.

[23] S. Knox, J. Harris, L. Calton, A.M. Wallace, A simple automated solid-phase extraction procedure for measurement of 25-hydroxyvitamin D3 and D2 by liquid chromatographytandem mass spectrometry, Ann Clin Biochem 46(Pt 3) (2009) 226-30.

[24] J.E. Compston, Sex steroids and bone, Physiol Rev 81(1) (2001) 419-447.

[25] M. Papageorgiou, D. Martin, H. Colgan, S. Cooper, J.P. Greeves, J.C.Y. Tang, W.D. Fraser, K.J. Elliott-Sale, C. Sale, Bone metabolic responses to low energy availability achieved by diet or exercise in active eumenorrheic women, Bone 114 (2018) 181-188.

[26] J. Upadhyay, O.M. Farr, C.S. Mantzoros, The role of leptin in regulating bone metabolism, Metabolism 64(1) (2015) 105-13.

[27] M.J. De Souza, S.L. West, S.A. Jamal, G.A. Hawker, C.M. Gundberg, N.I. Williams, The presence of both an energy deficiency and estrogen deficiency exacerbate alterations of bone metabolism in exercising women, Bone 43(1) (2008) 140-148. 
[28] B.C. Nindl, B.R. Barnes, J.A. Alemany, P.N. Frykman, R.L. Shippee, K.E. Friedl, Physiological Consequences of U.S. Army Ranger Training, Med Sci Sports Exerc 39(8) (2007) 1380-7.

[29] J.P. Casez, S. Fischer, E. Stussi, H. Stalder, A. Gerber, P.D. Delmas, J.P. Colombo, P. Jaeger, Bone mass at lumbar spine and tibia in young males--impact of physical fitness, exercise, and anthropometric parameters: a prospective study in a cohort of military recruits, Bone 17(3) (1995) 211-9.

[30] K.A. O'Brien, R.D. Pollock, M. Stroud, R.J. Lambert, A. Kumar, R.A. Atkinson, D.A. Green, A. Anton-Solanas, L.M. Edwards, S.D.R. Harridge, Human physiological and metabolic responses to an attempted winter crossing of Antarctica: the effects of prolonged hypobaric hypoxia, Physiol Rep 6(5) (2018).

[31] L. Vico, B. van Rietbergen, N. Vilayphiou, M.T. Linossier, H. Locrelle, M. Normand, M. Zouch, M. Gerbaix, N. Bonnet, V. Novikov, T. Thomas, G. Vassilieva, Cortical and Trabecular Bone Microstructure Did Not Recover at Weight-Bearing Skeletal Sites and Progressively Deteriorated at Non-Weight-Bearing Sites During the Year Following International Space Station Missions, J Bone Miner Res 32(10) (2017) 2010-2021.

[32] N.E. Andersen, J.P. Karl, S.J. Cable, K.W. Williams, J.C. Rood, A.J. Young, H.R. Lieberman, J.P. McClung, Vitamin D status in female military personnel during combat training, J Int Soc Sports Nut 7 (2010) 38.

[33] S. Iuliano-Burns, J. Ayton, S. Hillam, G. Jones, K. King, S. Macleod, E. Seeman, Skeletal and hormonal responses to vitamin D supplementation during sunlight deprivation in Antarctic expeditioners, Osteoporos Int 23(10) (2012) 2461-7.

[34] S. Iuliano-Burns, X.F. Wang, J. Ayton, G. Jones, E. Seeman, Skeletal and hormonal responses to sunlight deprivation in Antarctic expeditioners, Osteoporos Int 20(9) (2009) $1523-8$. 
[35] J.D. Schipilow, H.M. Macdonald, A.M. Liphardt, M. Kan, S.K. Boyd, Bone microarchitecture, estimated bone strength, and the muscle-bone interaction in elite athletes: an HR-pQCT study, Bone 56(2) (2013) 281-9.

[36] R.K. Evans, C.H. Negus, A.J. Centi, B.A. Spiering, W.J. Kraemer, B.C. Nindl, Peripheral QCT sector analysis reveals early exercise-induced increases in tibial bone mineral density, J Musculoskelet Neuronal Interact 12(3) (2012) 155-64.

[37] I. Varley, D.C. Hughes, J.P. Greeves, W.D. Fraser, C. Sale, Increased Training Volume Improves Bone Density and Cortical Area in Adolescent Football Players, Int J Sports Med 38(5) (2017) 341-346.

[38] R.M. Izard, W.D. Fraser, C. Negus, C. Sale, J.P. Greeves, Increased density and periosteal expansion of the tibia in young adult men following short-term arduous training, Bone 88 (2016) 13-9.

[39] E. Gaffney-Stomberg, L.J. Lutz, J.C. Rood, S.J. Cable, S.M. Pasiakos, A.J. Young, J.P. McClung, Calcium and vitamin D supplementation maintains parathyroid hormone and improves bone density during initial military training: a randomized, double-blind, placebo controlled trial, Bone 68 (2014) 46-56.

[40] T.J. O'Leary, S.C. Saunders, S.J. McGuire, M.C. Venables, R.M. Izard, Sex Differences in Training Loads during British Army Basic Training, Med Sci Sports Exerc 50(12) (2018) 2565-74.

[41] S.D. Blacker, D.M. Wilkinson, M.P. Rayson, Gender differences in the physical demands of British Army recruit training, Mil Med 174(8) (2009) 811-6.

[42] V.L. Richmond, J.M. Carter, D.M. Wilkinson, F.E. Homer, M.P. Rayson, A. Wright, J.L. Bilzon, Comparison of the physical demands of single-sex training for male and female recruits in the British Army, Mil Med 177(6) (2012) 709-15. 
[43] T.J. O'Leary, S.C. Saunders, S.J. McGuire, R.M. Izard, Sex differences in neuromuscular fatigability in response to load carriage in the field in British Army recruits, $\mathbf{J}$ Sci Med Sport 21(6) (2018) 591-595.

[44] H.M. Rice, S.C. Saunders, S.J. McGuire, T.J. O'Leary, R.M. Izard, Estimates of Tibial Shock Magnitude in Men and Women at the Start and End of a Military Drill Training Program, Mil Med (2018).

[45] P.J. Carden, R.M. Izard, J.P. Greeves, J.P. Lake, S.P. Myers, Force and acceleration characteristics of military foot drill: implications for injury risk in recruits, BMJ Open Sport Exerc Med 0(e000025) (2015) 1-7. 